\title{
Inter-relação bidirecional entre gestação e doença periodontal: revisão de literatura
}

\author{
Bidirectional interrelationship between pregnancy and periodontal disease: literature review \\ Interrelación bidireccional entre el embarazo y la enfermedad periodontal: revisión de la literatura
}

Recebido: 13/10/2021 | Revisado: 22/10/2021 | Aceito: 27/10/2021 | Publicado: 30/10/2021

\author{
Bianca Lopes Rigueira Nasser \\ ORCID: https://orcid.org/0000-0001-5415-3465 \\ Centro Universitário de Patos de Minas, Brasil \\ E-mail: biancalmr@unipam.edu.br \\ Dayviddy Lucas Magalhães Silva \\ ORCID: https://orcid.org/0000-0002-6708-2659 \\ Centro Universitário de Patos de Minas, Brasil \\ E-mail: dayviddylms@unipam.edu.br \\ Laura Cesário Oliveira \\ ORCID: https://orcid.org/0000-0002-7689-5061 \\ Centro Universitário de Patos de Minas, Brasil \\ E-mail: lauracesario@unipam.edu.br \\ Carolina Silva Pereira \\ ORCID: https://orcid.org/0000-0003-2020-3932 \\ Centro Universitário de Patos de Minas, Brasil \\ E-mail:carolinasp@unipam.edu.br \\ Thiago de Amorim Carvalho \\ ORCID: https://orcid.org/0000-0003-1153-0931 \\ Centro Universitário de Patos de Minas, Brasil \\ E-mail: thiagocarvalho@unipam.edu.br \\ Daniela Navarro Ribeiro Teixeira \\ ORCID: http://orcid.org/0000-0001-5485-8107 \\ Centro Universitário de Patos de Minas, Brasil \\ E-mail: danielanrteixeira@unipam.edu.br \\ Fabrício Campos Machado \\ ORCID: https://orcid.org/0000-0003-4603-8795 \\ Centro Universitário de Patos de Minas, Brasil \\ E-mail: fabriciocampos@unipam.edu.br
}

\begin{abstract}
Resumo
Os problemas que englobam a saúde bucal, incluindo a doença periodontal, têm sido cada vez mais reconhecidos como importantes precursores de consequências negativas no desempenho de atividades diárias e na qualidade de vida dos indivíduos. Esse fato se torna ainda mais relevante quando se trata da saúde de mulheres gestantes e do feto em desenvolvimento que pode nascer prematuramente ou com baixo peso. O objetivo deste trabalho é avaliar a interrelação entre a doença periodontal e a gravidez caracterizando sua associação com o pré-natal odontológico e a saúde do bebê. Trata-se de uma revisão narrativa da literatura, visando identificar quais os fatores associados à odontologia $\mathrm{e}$ ao pré-natal odontológico. Como critérios de inclusão foram utilizados artigos publicados nos últimos dez anos e disponibilizados em inglês e português empregando as palavras chaves odontologia e gravidez; odontologia e prénatal odontológico; odontologia e pré-natal e doenças periodontais. Concluiu-se que a saúde bucal materna influencia diretamente na sua própria saúde como um todo, mas também na saúde do bebê. Assim, é notória a importância de se realizar o pré-natal odontológico para prevenção de patologias bucais e sistêmicas, além de também evitar partos prematuros e com baixo peso ao nascer.
\end{abstract}

Palavras-chave: Assistência pré-natal; Assistência odontológica; Gestação; Doença periodontal.

\section{Abstract}

Problems involving oral health, including periodontal disease, have been increasingly recognized as important precursors of negative consequences on the performance of daily activities and on the quality of life of individuals. This fact becomes even more relevant when it comes to the health of pregnant women and the developing fetus that may be born prematurely or with low birth weight. The objective of this review is to evaluate the interrelationship between periodontal disease and pregnancy, characterizing its association with dental prenatal care and baby's health. This is a narrative review of the literature, aiming to identify the factors associated with dentistry and dental prenatal care. Articles published in the last ten years and available in English and Portuguese were included using the keywords dentistry and pregnancy; dentistry and dental prenatal care; dentistry and prenatal and periodontal diseases. It was concluded that maternal oral health influences directly their own health, but also the baby's health. Thus, the 
importance of performing dental prenatal care to prevent oral and systemic pathologies is notorious, as well as preventing premature and low birth weight deliveries.

Keywords: Prenatal care; Dental care; Pregnancy; Periodontal diseases.

\section{Resumen}

Los problemas que engloban la salud bucodental, incluida la enfermedad periodontal, han sido reconocidos cada vez más como importantes precursores de consecuencias negativas en el desempeño de las actividades diarias y la calidad de vida de las personas. Este hecho adquiere mayor relevancia cuando se trata de la salud de las mujeres embarazadas y del feto en desarrollo que puede nacer prematuramente o con bajo peso. El objetivo de este estudio es evaluar la interrelación entre la enfermedad periodontal y el embarazo, caracterizando su asociación con el cuidado prenatal dental y la salud del bebé. Se trata de una revisión narrativa de la literatura, con el objetivo de identificar qué factores se asocian a la atención prenatal dental y odontológica. Como criterio de inclusión, se utilizaron artículos publicados en los últimos diez años y disponibles en inglés y portugués, empleando las palabras clave odontología y embarazo; odontología y prenatal dental; odontología y prenatal y enfermedades periodontales. Se llegó a la conclusión de que la salud bucodental de la madre influye directamente en su propia salud en general, pero también en la del bebé. Así, es notoria la importancia de realizar el control prenatal dental para la prevención de patologías orales y sistémicas, además de evitar también los nacimientos prematuros y el bajo peso al nacer.

Palabras clave: Atención prenatal; Cuidado dental; Embarazo; Enfermedad periodontal.

\section{Introdução}

Os problemas que englobam a saúde bucal têm sido cada vez mais reconhecidos como importantes precursores de consequências negativas no desempenho de atividades diárias e na qualidade de vida dos indivíduos e da sociedade em que estão inseridos (Silva et al., 2020). De acordo com o relatório da Organização Mundial da Saúde (OMS) as doenças bucais causam dor, sofrimento, constrangimentos psicológicos e privações sociais, ocasionando inúmeros danos à qualidade de vida dessas pessoas (da Silva et al., 2018).

A conscientização de manter a higiene oral adequada e promover rapidez do tratamento de várias condições orais têm um efeito positivo, visto que o processo de prevenção gera inúmeros benefícios (Silva et al., 2020). Diante disso, é importante ressaltar a necessidade de uma atenção especial quando se trata da saúde oral das mulheres, o que se explica pela presença de diferentes estados fisiológicos, como a puberdade, a gravidez e a menopausa (Ouanounou \& Haas, 2016). Tais fatos devem ser levados em consideração, uma vez que essas condições são capazes de impactar de modo geral no estado de saúde das mulheres (Ouanounou \& Haas, 2016; Silva et al., 2020).

A gestação tem papel de destaque quando se consideram tais aspectos e isso se justifica pelo fato de que a gravidez traz consigo uma variedade de mudanças no corpo da mulher. Nesta fase, observa-se um aumento progressivo da secreção de hormônios que podem resultar em uma mudança na saúde geral das futuras mães (Marla et al., 2018). Diante disso, o acompanhamento multidisciplinar é essencial, já que a mulher nessa fase está receptiva a adquirir informações e mudar hábitos com o objetivo de preservar a sua qualidade de vida e a do bebê. Dessa forma, pode-se construir uma nova realidade que desmantela as crenças e desinformações por parte das gestantes e dos profissionais da saúde a respeito da importância do tratamento e da prevenção odontológica para a diminuição de riscos durante a gestação (Silva et al., 2020).

As mudanças que podem estar presentes durante a gestação envolvem disfunções sistêmicas, como as alterações cardiovasculares, hematológicas, respiratória, renal, gastrointestinal, endócrino e sistemas geniturinários e também consequências encontradas na cavidade oral, que podem ser localizadas tanto nos tecidos duros quanto nos tecidos moles (Cabral, et al., 2013). A doença periodontal é muito prevalente nessa fase, englobando a gengivite e a periodontite, que estão diretamente relacionadas às alterações no nível dos hormônios estrógeno e progesterona (Marla, et al., 2018). Pode-se ainda, encontrar um crescimento reativo chamado granuloma piogênico que frequentemente é diagnosticado na cavidade oral de mulheres grávidas, especialmente na gengiva inserida (Ouanounou \& Haas, 2016). A incidência de cárie dentária e biocorrosão dental endógena também têm um aumento significativo nessa etapa, justificado pelos frequentes episódios de náuseas e vômitos que alteram o pH da cavidade bucal (Marla, et al., 2018). 
Fica claro que na saúde materna, que engloba a saúde da criança, da mulher e o planejamento familiar, o cuidado pré-natal odontológico é um elemento essencial para garantir o sucesso da gestação. Isso porque a não realização de tais exames podem causar grandes danos à mãe e também ao feto em desenvolvimento, ocasionando assim, partos prematuros ou bebês de baixo peso ao nascer (Silva et al., 2020). Esse fato é considerado um problema de saúde pública e o Instituto Brasileiro de Geografia e Estatística (IBGE) aponta que a taxa de baixo peso ao nascer tem aumentado numa proporção de 1,2\% ao ano, principalmente em regiões menos desenvolvidas do país (Mascarenhas, et al., 2012).

$\mathrm{O}$ atendimento odontológico durante o período de gestação é um assunto bastante controverso, principalmente em função dos mitos que são baseados em crenças antigas sem fundamentação científica, tanto por parte da gestante quanto por parte dos cirurgiões-dentistas que não se sentem seguros ao atendê-las (Cabral, et al., 2013). O conhecimento dos cirurgiõesdentistas sobre os trimestres na gravidez é importante para mensurar e poder prever possíveis problemas, possibilitando cuidados odontológicos na prescrição de medicamentos e exames radiográficos, induzindo a um tratamento seguro, eficaz e com menor risco de efeitos deletérios aos bebês (Manrique-Corredor et al., 2019).

Dito isso, o objetivo desse estudo é identificar a inter-relação bidirecional entre aspectos periodontais e a gravidez, demonstrando sua influência sobre o bebê, e em outra face enfatizar a importância do pré natal odontológico, o articulando a temática e dando assim um contexto geral acerca do assunto.

\section{Metodologia}

Trata-se de uma revisão narrativa da literatura. De acordo com Rother (2007), são estudos que do ponto de vista teórico ou conceitual realizam uma revisão ampla a fim de descrever e discutir o desenvolvimento ou o "estado da arte". Assim, esta pode contribuir para o debate de determinadas temáticas, levantamento de questões e colaborar para a aquisição e atualização de conhecimentos em um curto espaço de tempo.

As buscas foram desenvolvidas entre os meses de Fevereiro e Junho de 2021, permitindo uma síntese sobre o assunto que engloba conhecimento científico, contribuindo para a prática na saúde baseada em evidências. Para a realização do estudo foi necessário a identificação do tema e seleção da questão norteadora; estabelecimento de critérios para inclusão e exclusão dos estudos; definição das informações a serem extraídas dos estudos selecionados e categorização desses; avaliação metodológica dos estudos incluídos; interpretação dos resultados; apresentação da revisão e síntese do conhecimento. As buscas foram desenvolvidas nas seguintes bases de dados: Literatura Latino-Americana e do Caribe em Ciências da Saúde (Lilacs), Pub Med, Scientific Electronic Library Online (Scielo) e Banco de Dados em Enfermagem Bibliografia Brasileira (BDEnf) via Biblioteca Virtual em Saúde (BVS).

Identificou-se quais os fatores associados à odontologia e o pré-natal odontológico assim como sua inter-relação com a doença periodontal. Com base na seleção dos artigos foram necessário dois revisores de forma independente, sendo realizada a leitura dos títulos, dos resumos e do material completo. Usou-se os seguintes descritores: "gestação", "pré-natal odontológico" e "doença periodontal". Foram critérios de inclusão artigos publicados dentro do período de 10 anos e disponibilizados em inglês e português. Na busca dos artigos foram utilizados descritores indexados aos Descritores de Ciência e Saúde (DeCS), e a estratégia de busca utilizada foi: odontologia e gravidez; odontologia e pré-natal odontológico; odontologia e pré-natal e doenças periodontais.

A análise crítica dos estudos selecionados e a síntese dos dados bibliográficos foram realizadas de forma descritiva, agrupados por temas pertinentes ao assunto estudado para facilitar a compreensão da evolução do conhecimento na área de saúde bucal das gestantes, que estão atreladas com a importância do pré-natal nessa fase. Assim, sendo possível observar, descrever e classificar os dados, com a intenção de colaborar com o processo de busca da temática principal deste trabalho. 
Os custos desembolsados ao estudo foram para fins de material de papelaria e internet necessário para seu desenvolvimento. O trabalho possuiu financiamento próprio dos pesquisadores.

\section{Resultados e Discussão}

Os resultados encontrados permitiram apresentar as relações em três categorias temáticas que nortearam a pesquisa: a significativa contribuição do pré-natal odontológico nessa fase da vida da mulher; a relação que a gravidez tem com a doença periodontal; e a prematuridade do bebê na presença da doença periodontal.

\subsection{A significativa contribuição do pré-natal odontológico nessa fase da vida da mulher}

O acesso à assistência odontológica na gravidez se torna algo relevante, devido ao fato de que o aconselhamento somado aos cuidados preventivos e educativos pode transformar uma realidade e precaver a mulher gestante de vários problemas de ordem geral e bucal, sendo que a ausência de tal assistência pode não evidenciar e prevenir tais condições (Matsubara \& Demetrio, 2016).

Torna-se uma afirmativa de que a saúde se inicia pela cavidade oral, e tendo em vista que a gravidez traz consigo inúmeras alterações fisiológicas, consequentemente trará à cavidade oral (Degasperi et al., 2021). No entanto, é importante a conscientização das gestantes de que as mudanças biológicas, hormonais e psicológicas ocorridas desde o início da gestação fazem com que fiquem mais suscetíveis às doenças orais, necessitando de um maior cuidado nesse aspecto, assegurando o desenvolvimento normal da gestação, permitindo o parto de um recém-nascido saudável, sem impacto para a saúde materna. (Degasperi et al., 2021, Moura et al., 2020).

Quando se trata da prevenção da saúde bucal, o controle do biofilme assume um importante papel e dentro dos possíveis tratamentos está a profilaxia dentária, raspagem e alisamento radicular (Matsubara \& Demetrio, 2016). Dessa forma, é imprescindível que esse acompanhamento seja feito com periodicidade nos 3 trimestres de gestação para que, a partir dessas intervenções, seja possível remover os focos de infecção e não causar danos ao feto e à mãe (Matsubara \& Demetrio, 2016).

No período gestacional, a maioria dos procedimentos odontológicos podem ser realizados. Porém, devem ser tomados algumas medidas especiais como planejar sessões curtas, adequar a posição da cadeira e evitar consultas matinais, já que neste período as gestantes têm mais episódios de ânsia de vômito e risco de hipoglicemia (Moreira et al., 2015). Fazer uma anamnese detalhada, ter uma boa comunicação com o médico da gestante, avaliar o estado de saúde geral da mesma são passos que devem ser seguidos com critério, pois são decisivos previamente a qualquer procedimento odontológico (Matsubara\&Demetrio, 2016).

O mais recomendado é que se faça os procedimentos odontológicos no segundo trimestre, isso porque, durante esse período, a organogênese está completa e o feto já está mais desenvolvido e também devido ao fato de que a mãe se sente mais confortável do que nos estágios iniciais ou finais de sua gravidez (Botelho et al., 2019). No primeiro trimestre (primeiros 90 dias de gestação), o embrião ainda está em processo de organogênese e nesse período o feto se encontra mais susceptível à teratogênese quando se torna necessário a prescrição de medicamentos (Ouanounou \& Haas, 2016).

Durante o atendimento clínico, o cirurgião dentista (CD) deve salientar-se para manter a paciente em posição de decúbito lateral (deitada de lado) para evitar o surgimento de complicações como hipotensão, taquicardia e síncope, redução da circulação útero-placentária, representando perigo para o feto (Bi et al., 2019; Caneppele et al., 2011). Independentemente do período gestacional, as consultas prolongadas devem ser evitadas devido à possibilidade de ocorrer hipotensão supina ou síndrome da veia cava especialmente na segunda metade da gravidez, quando o útero já se apresenta com o volume bastante aumentado, podendo comprimir a artéria aorta e veia cava se a paciente for mantida por muito tempo em posição de decúbito dorsal (Caneppele et al., 2011). 
Tendo em vista os procedimentos realizados nas consultas odontológicas, seus cuidados especiais devem ser lembrados (Ouanounou \& Haas, 2016). Tem-se uma preocupação maior na prescrição de medicamentos às grávidas devido aos potenciais efeitos teratogênicos que alguns medicamentos apresentam, uma vez que esses medicamentos podem atravessar a placenta e afetar o desenvolvimento normal do feto (Ouanounou \& Haas, 2016).

Geralmente os medicamentos precisam de modificações na dosagem durante a gestação, devendo o cirurgião dentista estar atento à posologia e à indicação para o tratamento medicamentoso (Ouanounou \& Haas, 2016). Desta maneira, visando sempre à melhor conduta, o profissional deve conhecer os medicamentos que podem ser prescritos para tais pacientes, as possíveis consequências que esse tratamento pode ocasionar à mulher grávida, além de sua eficácia e segurança (Amadei et al., 2011). O princípio que norteia a escolha da terapêutica a ser utilizada para gestantes é baseado nos riscos-benefícios para o feto e a mãe (Amadei et al., 2011).

A agência americana Food and Drug Administration (FDA) classificou os fármacos quanto aos efeitos na gestação em categorias de risco A, B, C, D e X (Amadei et al., 2011). Os medicamentos que estão incluídos nos grupos A e B são considerados seguros (Amadei et al., 2011). Os do grupo C são aqueles que em amostras tiveram efeitos adversos em animais, mas não possuem comprovações em humanos (Amadei et al., 2011). Já os medicamentos na categoria D são aqueles que devem ser evitados, pois há efeitos teratogênicos comprovados em humanos (Amadei et al., 2011). Por fim, os que estão incluídos no grupo X, são medicamentos contraindicados devido aos seus efeitos de anormalidade no desenvolvimento fetal (Ouanounou \& Haas, 2016).

O uso de anestésicos locais em gestantes é considerado seguro, não existindo contraindicações no seu uso regular (Amadei et al., 2011). Porém, as doses necessárias para bloqueio nervoso e para produzir toxicidade são reduzidas, não se sabendo ao certo se esse efeito é devido aos níveis elevados de estrogênio, à elevação de progesterona ou a algum outro fator (Ouanounou \&Haas, 2016). Portanto, o uso da adrenalina se torna importante no anestésico de escolha, pois contribuirá para a diminuição do sangramento no local de administração e aumento da duração do efeito anestésico, sendo vantajoso tanto para o dentista quando para a gestante (Ouanounou \& Haas, 2016).

De maneira geral, os anestésicos são considerados seguros (Ouanounou \&Haas, 2016). A primeira escolha é normalmente a Lidocaína a 2\% com Epinefrina 1;100.000 (categoria B), devendo-se utilizar no máximo dois tubetes (3,6 ml) por sessão de atendimento (Amadei et al., 2011). Não devem ser administrados benzocaína, mepivacaína, bupivacaína e articaína (categoria C), uma vez que estes fármacos diminuem a circulação placentária e apresentam o risco de metemoglobinemia e hipóxia fetal (Amadei et al., 2011). Na administração dos anestésicos locais, deve-se adotar como cuidado a injeção lenta da solução com aspiração prévia, evitando a injeção intravascular (Matsubara \& Demetrio, 2016). A prilocaína também está contraindicada e vasoconstritores como felipressina e ocitocina também, pois podem levar a contrações uterinas (Amadei et al., 2011).

Com relação aos analgésicos, o mais indicado em geral pelos cirurgiões dentistas é o paracetamol (classificação FDA de B) (Dôrico®, Tylenol®), podendo ser prescrito também a pacientes gestantes (Amadei et al., 2011). Estudos recentes têm associado o uso desse medicamento com o risco de transtorno de déficit de atenção e hiperatividade no recém-nascido, porém conclusões definitivas não foram tiradas (Ouanounou \& Haas, 2016). A dipirona sódica (Novalgina®) é o analgésico de segunda escolha, pois pode provocar agranulocitose, ou seja, a redução do número de granulócitos no sangue periférico (neutropenia), podendo predispor o indivíduo às infecções (Amadei et al., 2011).

Comumente na odontologia se faz uso dos antiinflamatórios não esteroidais (AINEs), que inclui o ibuprofeno e naproxeno (Ouanounou \&Haas, 2016). Na gravidez, tais medicamentos podem não ser tão vantajos, pois o ibuprofeno recebe classificação de Categoria B no primeiro e segundo trimestre, e no terceiro ele recebe a categoria D, não devendo ser administrado (Ouanounou \&Haas, 2016) . Isso se deve ao fato de que o uso de AINEs no final da gravidez pode prolongar a 
duração do trabalho de parto, aumentando o sangramento durante o parto e o fechamento prematuro do canal arterial (Ouanounou \&Haas, 2016).

Nos tratamentos odontológicos é comum o uso dos ansiolíticos, pois os pacientes podem ter algum receio e ansiedade no momento da consulta. Dessa forma, para as gestantes, é necessário um cuidado especial (Ouanounou \&Haas,

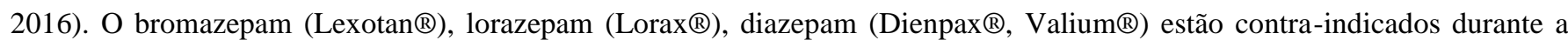
gravidez, pois se suspeita que estes medicamentos tenham um poder teratogênico razoável (Amadei et al., 2011). Portanto, nos casos de ansiedade considerável, deve-se realizar a sedação com o óxido nitroso (NO) ou benzodiazepinicos, que apesar de não ter uma classificação no FDA, são aceitos (Ouanounou \& Haas, 2016).

Com relação aos antibióticos, os mais utilizados na prática odontológica são seguros nesse período, mas o cirurgião dentista deve considerar cuidadosamente o risco benefício de cada um (Amadei et al., 2011). A estreptomicina tem sido associada à lesão do oitavo par craniano e defeitos esqueléticos no feto (Ouanounou \& Haas, 2016; Amadei et al., 2011). Deve-se tomar um cuidado maior com as tetraciclinas (classificados na categoria D), pois quando administradas até a segunda metade da gravidez causam hipoplasia dos dentes e dos ossos do feto e foram associadas também à ocorrência de cataratas congênitas (Ouanounou \& Haas, 2016; Amadei et al., 2011). A primeira opção em caso de infecção é a amoxicilina e a penicilina que estão na categoria B (Ouanounou \& Haas, 2016). Caso o paciente apresente um quadro alérgico à penicilina, a clindamicina pode ser administrada, uma vez que esta também se inclui na categoria B (Ouanounou \& Haas, 2016). A eritromicina, apesar de estar também na categoria que se permite o uso de grávidas, deve ser evitada, pois está relacionada à hepatite colestática (Ouanounou \& Haas, 2016; Amadei et al., 2011)

As tomadas radiográficas devem ser realizadas quando realmente necessário, em qualquer trimestre da gestação (Caneppele et al., 2011). Em casos de necessidade, o cirurgião dentista deve se atentar à alguns cuidados que são imprescindíveis: evitar radiografias desnecessárias, proteger o abdome com avental de chumbo, evitar repetições por erro de técnica, evitar ângulos direcionados para o abdome, usar filmes rápidos e pequenos tempos de exposição (Caneppele et al., 2011). Sendo executado desse modo, esse procedimento não atingirá o desenvolvimento fetal (Caneppele et al., 2011). Para existir a possibilidade de má-formação ou aborto espontâneo, é necessária uma exposição de 5 rads (Caneppele et al., 2011). Uma tomada radiográfica intrabucal equivale a 0,01 milirads de radiação, menos que a radiação cósmica adquirida diariamente, demonstrando portanto sua segurança, desde que realizada da forma correta (Caneppele et al., 2011).

\subsection{A inter-relação entre gravidez e doença periodontal}

A gestação é um momento em que a saúde bucal deve ser acompanhada com muito cuidado, já que, neste período, as mulheres estão mais susceptíveis a algumas anormalidades como hiperemia, edema e grande tendência ao sangramento gengival (Manrique-Corredor et al., 2019; Mascarenhas et al., 2012). Um programa de atenção à saúde oral deve ser incluído logo quando a gestante chega para um acompanhamento pré-natal (Manrique-Corredor et al., 2019). Nessa fase deve haver uma maior motivação e incentivo para avaliar seu estado de saúde bucal e possibilidade de tratamento para doença periodontal durante a gestação para que assim, evite consequências graves que alguns problemas bucais tendem a ocasionar (ManriqueCorredor et al., 2019)

O estrógeno e a progesterona, são hormônios sexuais que durante a gestação elevam drasticamente seus níveis, estes favorecem o suporte nutricional para os microrganismos da cavidade oral, desse modo, bactérias acidogênicas, como o Streptococos sp, se desenvolvem facilmente, no entanto, a acidificação da cavidade oral, associada à queda do pH, uma alimentação rica em carboidratos, e descontrole da placa bacteriana ou biofilme, resultados de uma precária higienização bucal, são condições que incrementam a predisposição para que a gestante desenvolve doença cárie e também as doenças gengivais como gengivite e periodontite (Calvi et al., 2020, Degasperi et al., 2021). 
A doença periodontal é a principal complicação relacionada aos problemas na gestação, pois se torna um reservatório para a disseminação hematogênica de bactérias orais e consequentemente a produção de mediadores inflamatórios que vão agir diretamente na unidade materno-fetal (Armitage, 2013). Detectada a presença dessa comorbidade, a circulação desses mediadores (tais como a interleucina) se torna relevante (Bi et al., 2019). Esse fato se concretiza em resposta à infecção, e a partir disso pode-se ter um efeito deletério até mesmo para órgãos distantes, como o útero, o que garante a relação entre periodontite e o nascimento prematuro (Armitage, 2013).

O brusco aumento desses hormônios femininos circulantes durante a gestação é responsável pela exacerbação da reação inflamatória gengival, principalmente por sua ação vasodilatadora e diminuição da resposta imune e à cicatrização das feridas. (Dave et al., 2021; Mascarenhas et al., 2012). Os níveis desses hormônios ovarianos elevam-se ainda mais no oitavo mês de gravidez (Bi et al., 2019). Neste instante, teremos severa inflamação gengival por ação hormonal (Mascarenhas et al., 2012).

O estrógeno predispõe as alterações vasculares, e a progesterona atua estimulando a produção de mediadores inflamatórios e esses hormônios possuem receptores no tecido gengival, fato que ocasiona maior acúmulo desses hormônios no interior desse tecido (Spezzia, 2016). Estes podem agir influenciando de várias formas: modificando a resposta dos tecidos periodontais, advinda da ação do biofilme dental, na constituição das colônias de bactérias presentes no biofilme dental e/ou estimulando a produção de prostaglandinas (Spezzia, 2016).

Quando os agentes causadores desta infecção não são removidos periodicamente, tendem a atingir o tecido de suporte dos dentes (osso alveolar) e estimular sua reabsorção por meio de reação inflamatória ((Dave et al., 2021, Mascarenhas et al., 2012). Nas bolsas periodontais podem ser isoladas diversas espécies bacterianas, muitas delas gram-negativas e algumas se caracterizando por alta patogenicidade, são elas: Actinobacillus, Bacteróides e Porphyromonas (Bi et al., 2019). Quando o organismo humano reconhece a presença destas bactérias, inicia-se a reação de imunidade inata, primeira linha de defesa contra os agentes agressores (Mascarenhas et al., 2012).

\subsection{A prematuridade do bebê na presença da doença periodontal}

O parto prematuro, classificado como aquele cujo nascimento antecede 37 semanas de gestação, é a principal causa de recém-nascidos com baixo peso (essa classificação se enquadra quando o bebê nasce pesando menos que 2,500kg) (Manrique-Corredor et al., 2019). Esse fator é uma das principais causas das mortes perinatais que ocorrem em neonatos nascidos prematuramente (em sua maioria nascidos antes de 32 semanas de gestação) (Matsubara \& Demetrio, 2016). Deve-se salientar também que tais causas podem ocasionar morbidades neurológicas no neonato a curto prazo (Matsubara \& Demetrio, 2016).

A epidemiologia do parto pré-termo está relacionada a um foco infeccioso, porém esse isoladamente não é capaz de ativar a cascata inflamatória para a unidade feto-placentário (Silva et al., 2020). Esse fato se relaciona uma vez que os impulsos inflamatórios podem resultar numa hiperirritabilidade da musculatura lisa uterina, causando a contração prematura do útero e dilatação cervical, agindo como gatilho para o parto prematuro (da Cunha et al., 2015; Dave et al., 2021). As infecções e os processos inflamatórios resultantes podem causar danos à placenta, restringindo, dessa forma, o crescimento fetal (da Cunha et al., 2015).

Quando há um foco infeccioso durante a gravidez, ocorrem inúmeras respostas fisiológicas no organismo da mulher grávida em resposta a estímulo e desse modo, geram consequências também na unidade fetoplacentária, alterando a troca de nutrientes entre a mãe e o feto (Schenkein, 2012). O nascimento do bebê se dá então de forma prejudicial, fazendo com que ele nasça com baixo peso. (Bi et al., 2019; Schenkein, 2012). 
As citosinas inflamatórias produzidas no periodonto em resposta do foco infeccioso se tornam circulantes no sangue da mulher grávida e ativa mediadores inflamatórios, tais como a prostaglandina, pelo âmnio, criando um ambiente hostil para o feto (Armitage, 2013; Cabral et al., 2013). As infecções periodontais podem servir de reservatórios para os microorganismos, anaeróbios gram-negativos e de seus produtos, como lipopolissacarídeos (LPS) e endotoxinas, além de produzirem quantidade significativa de mediadores inflamatórios (Armitage, 2013). Tal fato se associa com a excessiva presença de líquido amniótico contendo IL-1 $\beta$ ou com uma perturbação da biodisponibilidade e/ou bio-resposta desta citosina, que é fundamental para a reação pró inflamatória contra estímulos infecciosos (Armitage, 2013, Silva et al., 2020).

Bi e colaboradores (2019), relataram em sua revisão sistemática e metanálise de estudo randomizado controlado (RCTs), que houve um risco reduzido de mortalidade perinatal (RR, 0,53) e parto prematuro $(\mathrm{PTB})(<37$ semanas) $(\mathrm{RR}, 0,78)$ quando a mulher foi submetida ao tratamento periodontal durante a gravidez, sem risco de malformação congênita. Porém, não houve mudanças nos riscos de muito PTB ( $<35$ ou $<32$ semanas), baixo peso ao nascer (BPN), muito BPN, pequeno para a idade gestacional (PIG), admissão na unidade de terapia intensiva neonatal (UTIN), comprimento do nascimento, índice de Apgar, cesariana, pré-eclâmpsia ou diabetes gestacional (Bi et al., 2019).

Conclui-se por meio do exposto, que o pré-natal tem uma grande significância quando se trata da prevenção de problemas que podem ser consequências da gravidez, devido às alterações ocasionadas neste processo (Degasperi et al., 2021). Isso se torna relevante, pois para a evolução da gestação de sucesso necessita de um acompanhamento profissional especializado para que haja o estabelecimento de uma comunicação eficaz com as gestantes e seus familiares. (Degasperi et al., 2021). Diante disso, em 2000, no no Brasil, foi criado o Programa de Humanização do Parto e do Nascimento (PHPN), que implementou um protocolo de ações para diminuir a mortalidade materna e perinatal e prevenir futuras complicações (Degasperi et al., 2021). Nesse instante foi primordial favorecer e promover o início precoce do cuidado pré-natal, que envolveria os quesitos fisiológicos e odontológicos. (Degasperi et al., 2021, Moura et al., 2020).

\section{Considerações Finais}

Haja vista as alterações fisiológicas e transitórias, sistêmicas e bucais causadas pela gestação incluindo a presença da doença periodontal, este trabalho demonstra a importância do conhecimento desse quadro por parte dos cirurgiões dentistas. Esse conhecimento empodera o profissional para que ele possa desmistificar barreiras culturais que impedem a gestante de procurar o tratamento odontológico, colabora com o fomento do autocuidado apoiado, além de cooperar com a equipe multiprofissional de cuidados com a mulher grávida, objetivando a minimização dos riscos de parto prematuro e consequentemente o nascimento de bebês de baixo peso. Outra vertente de corroboração da necessidade do trabalho é enfatizar as consequências da não realização do pré-natal odontológico para o binômio mãe-bebê, bem como a forte influência que a mãe terá sobre seu filho, em relação à transmissão de hábitos de saúde bucal.

\section{Referências}

Amadei, S. U., Carmo, E. D. do, Pereira, A. C., Silveira, V. Á. S., \& Rocha, R. F. da. (2011). Prescrição medicamentosa no tratamento odontológico de grávidas e lactantes. Revista Gaúcha de Odontologia, 59(0), 31-37. http://revodonto.bvsalud.org/pdf/rgo/v59s1/a05v59s1.pdf

Armitage G. C. (2013). Bi-directional relationship between pregnancy and periodontal disease. Periodontology 2000, 61(1), 160-176. https://doi.org/10.1111/j.1600-0757.2011.00396.x

Botelho, D. L. L., Lima, V. G. A., Barros, M. M. A. F., \& Almeida, J. R. de S. (2019). Odontologia e gestação: A importância do pré-natal odontológico. Revista Sanare, 18(2), 69-77. https://sanare.emnuvens.com.br/sanare/article/view/1376/70

Bi, W. G., Emami, E., Luo, Z.-C., Santamaria, C., \& Wei, S. Q. (2019). Effect of periodontal treatment in pregnancy on perinatal outcomes: a systematic review and meta-analysis. The Journal of Maternal-Fetal \& Neonatal Medicine, 1-10. https://doi.org/10.1080/14767058.2019.1678142

Brasil (2018). A saúde bucal no Sistema Único de Saúde. Ministério da Saúde. Brasília- DF https://bvsms.saude.gov.br/bvs/publicacoes/saude_bucal_sistema_unico_saude.pdf 
Cabral, M. C. B., Santos, T. de S., \& Moreira, T. P. (2013). Percepcão das gestantes do Programa de Saúde da Família em relacão à saúde bucal no município de Ribeirópolis, Sergipe, Brasil. Revista Portuguesa de Saúde Pública, 31(2), 160-167. https://doi.org/http://dx.doi.org/10.1016/j.rpsp.2013.05.004

Caneppele, T. M. F., Yamamoto, E. C.,Valera, M. C., \& Araújo, M. A. M. de. (2011). Conhecimento dos cirurgiões-dentistas sobre o atendimento de pacientes especiais: hipertensos, diabéticos e gestantes. Journal of Biodentistry and Biomaterials, 1(1), 31-41. https://www.unibjournal.com.br/pdf/revista1-artigo4.pdf

Calvi, V.L., Chalub, L.O., Carmo, A.F.B., Levi, Y.L.A.S., Prado, R.L., Maia, L.P. (2020). Conhecimento dos alunos dos cursos de Odontologia e Medicina sobre a inter-relação entre doenças periodontais e doenças sistêmicas. Research, Society and Development, v. 9, n. 12. DOI: http://dx.doi.org/10.33448/rsdv9i12.10967

Da Cunha, A. J. L. A., Leite, Á. J. M., \& de Almeida, I. S. (2015). The pediatrician's role in thefirstthousanddays of thechild: thepursuit of healthynutrition and development. Jornal de Pediatria, 91(6), S44-S51. https://doi.org/10.1016/j.jped.2015.07.002

Da Silva, V.C., Santana,G.S., Queiroz, E.d.C., Martins, L.F.B. (2018). Doenças periodontais na gravidez: revisão de literatura. Encontro de Extensão, Docência e Iniciação Científica, 5(1), 1/4. http://publicacoesacademicas.unicatolicaquixada.edu.br/index.php/eedic/article/view/3116/0

Dave, B.H., Shah, B.E., Gaikwad, R.V., Shah, S.S. (2021). Association of preterm low-birth-weight infants and maternal periodontitis during pregnancy: An interventional study, v. 39, p. 183-188. DOI: 10.4103/jisppd.jisppd_270_20

Degasperi, J.U., Dias, A.J.W., Boleta-Ceranto, D.C.F. (2021). Alterações orais e sistêmicas decorrentes da gestação e a importância do pré-natal médico e odontológico para redução das complicações gestacionais. Research, Society and Development, v. 10, n. 3. DOI: http://dx.doi.org/10.33448/rsd-v10i3.12976

Manrique-Corredor, E. J., Orozco-Beltran, D., Lopez-Pineda, A., Quesada, J. A., Gil-Guillen, V. F., \& Carratala-Munuera, C. (2019). Maternal periodontitis and preterm birth: Systematic review and meta-analysis. Community Dentistry and Oral Epidemiology, 47(3), 243-251. https://doi.org/10.1111/cdoe.12450

Marla, V., Srii, R., Roy, D. K., \&Ajmera, H. (2018). The Importance of Oral Health duringPregnancy: A review. Medical Express, 5. https://doi.org/10.5935/medicalexpress.2018.mr.002

Mascarenhas, V. I., Vilarinho, L. A. L., Moura, L. de F. A. de D., Moura, M. S. de, \& Ferro, L. B. (2012). Correlação entre saúde periodontal e idade gestacional [Review of Correlação entre saúde periodontal e idade gestacional]. Revista de odontologia da UNESP, 41(6), 408-414. https://www.scielo.br/j/rounesp/a/xCKRFSg5xzCwK4pz5GdkWBP/?format=pdf\&lang=pt

Matsubara, A. S. \&Demetrio, A.T.W. (2017). Atendimento odontológico às gestantes: revisão da literatura. Revista UNINGÁ Review, 29(2), 42-47. https://www.mastereditora.com.br/periodico/20170204_122413.pdf

Moura, L.M., Araújo, A.A., Cerqueira, I.K.M., Steiner-Oliveira, C., Alves-Silva, E.G., Gomes, B.P.F.A, Almeida- Gomes, R.F. (2020). Conhecimento e atitude sobre a saúde bucal materno-infantil. Research, Society and Development, v. 9, n. 9. DOI: http://dx.doi.org/10.33448/rsd-v9i9.6969

Moreira, M.R., Santin, G.C., Matos, L.G., Gravina, D.B.L. (2015). Pré-natal odontológico: noções de interesse. Journal of Management and Primary Health Care, 6(1), 77-85. https://www.jmphc.com.br/jmphc/article/view/234/Pre-natal\%20odontologico

Ouanounou, A., \& Haas, DA (2016). Terapia medicamentosa na gravidez: implicações para a prática odontológica. British Dental Journal, 220 (8), $413-417$. doi: 10.1038 / sj.bdj.2016.299

Rother, E. T. (2007). Revisão sistemática X revisão narrativa. Acta Paulista de Enfermagem. 20(2). https://doi.org/10.1590/S0103-21002007000200001

Schenkein, H. A., Koertge, T. E., Sabatini, R., Brooks, C. N., \& Gunsolley, J. C. (2012). Birth weight of infants of mothers with aggressive periodontitis. Journal of periodontology, 83(3), 279-286. https://doi.org/10.1902/jop.2011.110192

Silva, C. C. da, Savian, C. M., Prevedello, B. P., Zamberlan, C., Dalpian, D. M., \& Santos, B. Z. dos. (2020). Acesso e utilização de serviços odontológicos por gestantes: revisão integrativa de literatura. Ciência \& Saúde Coletiva, 25(3), 827-835. https://doi.org/10.1590/1413-81232020253.01192018

Spezzia, S. (2016). Inter-relação entre hormônios sexuais e doenças periodontais nas mulheres / Interrelationshipbetween Sex Hormones and Periodontal Diseases in Women. Braz J Periodontol, 26(2), 40-47. http://www.revistasobrape.com.br/arquivos/2016/junho/REVPERIO_ JUNHO_2016_PUBL_SITE_PAG-40_A_47.pdf

Vasconcelos, R.G., Vasconcelos, M.G., Mafra, R.P., Júnior, L.C.A., Queiroz, L.M., Barboza, C.A.G. (2012). Atendimento odontológico a pacientes gestantes: como proceder com segurança. Revista Brasileira de Odontologia, 69(1), 120-124. http://revodonto.bvsalud.org/pdf/rbo/v69n1/a27v69n1.pdf 